
allemande

51-2 | 2019

Les Humanités environnementales : circulations et renouvellement des savoirs en France et en Allemagne

\title{
Quels savoirs de la « durabilité » en France et en Allemagne?
}

Jalons pour une mise en perspective comparée sous le regard des sciences sociales

Philippe Hamman

\section{(2) OpenEdition}

\section{Journals}

Édition électronique

URL : https://journals.openedition.org/allemagne/2017

DOI : $10.4000 /$ allemagne. 2017

ISSN : 2605-7913

Éditeur

Société d'études allemandes

Édition imprimée

Date de publication : 10 décembre 2019

Pagination : 425-450

ISSN : 0035-0974

Référence électronique

Philippe Hamman, «Quels savoirs de la « durabilité » en France et en Allemagne? », Revue d'Allemagne et des pays de langue allemande [En ligne], 51-2 | 2019, mis en ligne le 02 décembre 2020, consulté le 20 mai 2021. URL : http://journals.openedition.org/allemagne/2017 ; DOI : https://doi.org/10.4000/ allemagne.2017 


\section{Quels savoirs de la «durabilité» en France et en Allemagne? Jalons pour une mise en perspective comparée sous le regard des sciences sociales}

\section{- Philippe Hamman*}

\section{Introduction}

Participant d'un questionnement plus large des circulations au sein du champ des Humanités environnementales, cet article interroge les savoirs de la "durabilité » en France et en Allemagne, du point de vue des sciences sociales, afin d'esquisser une mise en perspective comparée. Nous nous fondons sur deux hypothèses:

(i) Lors du sommet de la Terre à Rio en 1992, le "développement durable» a été présenté comme une alternative en rupture avec les seuls impératifs de la croissance économique, tout en maintenant un flou appropriable par de nombreux acteurs aux objectifs différents $^{(1)}$. À partir de la scène internationale, et en se diffusant progressivement à différents échelons territoriaux, mettant en relation «global» et «local», il est ainsi devenu un répertoire à succès des décennies 1990-2000. Apparemment objet de consensus de la part d'une diversité d'acteurs sociaux: décideurs politiques et économiques, mais aussi associatifs environnementalistes, etc., sa pertinence pratique a alors été avérée ${ }^{(2)}$. Par ses effets sur les références de l'action publique et dans le monde social, il s'est également imposé comme un objet d'étude, voire un paradigme, dans les analyses de sciences sociales, tant en France qu'en Allemagne.

* Professeur de sociologie de la ville et de l'environnement, Institut d'urbanisme et d'aménagement régional, Faculté des sciences sociales, laboratoire Sociétés, acteurs, gouvernement en Europe (SAGE, UMR 7363), CNRS et Université de Strasbourg, phamman@unistra.fr.

1 Phil McManus, «Contested Terrains: Politics, Stories and Discourses of Sustainability», Environmental Politics, 5/1 (1996), p. 48-73; Edwin ZACCAI, Le développement durable. Dynamique et constitution d'un projet, Bruxelles, PIE-Peter Lang, 2002.

2 Cyria Emelianoff et Ruth Stegassy (dir.), Les pionniers de la ville durable. Récits d'acteurs, portraits de villes en Europe, Paris, Autrement, 2010; Philippe Hamman, Sociologie urbaine et développement durable, Bruxelles, De Boeck, 2012. 
(ii) Mais, depuis la fin des années 2000, la crise économique mondiale en a révélé les failles, opposant schématiquement une vision technique et économique de la durabilité à une version écologique et sociale ${ }^{(3)}$.

Qu'en est-il dès lors aujourd'hui? Repère-t-on des évolutions, voire des alternatives, au paradigme de la durabilité, et que peuvent-elles nous apprendre des circulations d'énoncés et de savoirs qui s'opèrent en France et en Allemagne?

\section{Dispositif méthodologique}

Méthodologiquement, considérant que la publication de travaux et les controverses académiques en sciences humaines et sociales sont des indicateurs valables, non seulement d'un «front de recherche» important, mais aussi de débats sociétaux, dont ils se font l'écho et qu'ils retraduisent ou mettent à distance, nous nous appuyons sur des lectures croisées d'ouvrages saillants allemands et français. Le statut de manuel ou de textbook, la visibilité des éditeurs en question et des auteurs/coordinateurs fournit un premier cadre de «représentativité»: l’inscription de tel énoncé ou notion dans ces opus peut être vue comme un mode de consécration dans l'univers scientifique, en même temps que le signe d'une certaine portée sociale et pratique ${ }^{(4)}$.

En ce sens, nous retenons, dans le cadre forcément limité de cet article, qui propose un regard franco-allemand:

- deux manuels francophones de sociologie de l'environnement: le Manuel de sociologie de l'environnement, somme collective (506 p.) publiée en 2012 sur l'initiative d'un comité éditorial issu du réseau thématique «Sociologie de l'environnement et des risques" de l'Association française de sociologie ${ }^{(5)}$ et la synthèse de Chantal Aspe et Marie Jacqué, Environnement et société. Une analyse sociologique de la question environnementale (272 p.), éditée la même année ${ }^{(6)}$. Ils sont mis en parallèle avec deux manuels germanophones: l'imposant Handbuch Umweltsoziologie (732 p.), coordonné par Matthias Groß et publié en $2011^{(7)}$, et le manuel devenu «classique» de Joseph Huber, Allgemeine Umweltsoziologie, dont la deuxième édition revue (353 p.)

3 Voir Edwin ZACCAI, «Le développement durable malmené», in: Rémi BARré, Thierry Lavoux et Vincent Piveteau (dir.), Un demi-siècle d'environnement entre science, politique et prospective. En l'honneur de Jacques Theys, Versailles, Quae, 2015, p. 119-129; Isabelle HaJEk et Philippe HaMMAN (dir.), La gouvernance de la ville durable entre déclin et réinventions. Une comparaison Nord/Sud, Rennes, Presses universitaires de Rennes, 2015.

4 Cette mobilisation d'ouvrages de synthèse fournit des jalons qui peuvent être approfondis par des études bibliométriques et lexicales, comme nous l'avons montré ailleurs: Philippe HAmman, Virginie Anquetin et Céline Monicolle, "Contemporary Meanings of the "Sustainable City". A Comparative Review of the French and English-Language Literature», Sustainable Development, 25/4 (2017), p. 336-355, et «Du "développement durable” à la "ville durable” : quels débats aujourd'hui? ", VertigO, $17 / 1$ (2017), en ligne: vertigo.revues.org/18466.

5 Rémi Barbier, Philippe Boudes, Jean-Paul Bozonnet, Jacqueline Candau, Michelle Dobré, Nathalie Lewis et Florence Rudolf (dir.), Manuel de sociologie de l'environnement, Québec, Presses de l’Université Laval, 2012.

6 Chantal Aspe et Marie JACQuÉ, Environnement et société. Une analyse sociologique de la question environnementale, Paris, Éditions de la Maison des Sciences de l'Homme/Versailles, Quae, 2012.

7 Matthias Groß (éd.), Handbuch Umweltsoziologie, Wiesbaden, VS Verlag, 2011. 
a elle aussi paru en $2011^{(8)}$. L'étude de ces ouvrages est complétée par les apports comparatifs offerts par le livre collectif Environnement et sciences sociales en France et en Allemagne, publié en 2014, dix ans après le colloque à son origine ${ }^{(9)}$;

- deux ouvrages dans chaque langue sur la durabilité en tant que telle, qui témoignent de la diffusion du registre au cours de la décennie 2000: côté français, l'important volume collectif (517 p.) dirigé par Bertrand Zuindeau, Développement durable et territoire, publié en $2010^{(10)}$, et celui coordonné par Josiane Stoessel-Ritz, Maurice Blanc et Nicole Mathieu, Développement durable, communautés et sociétés. Dynamiques socio-anthropologiques, publié en 2012 (230p.) ${ }^{(11)}$; côté allemand, le manuel d'Armin Grunwald et Jürgen Kopfmüller, Nachhaltigkeit, publié en 2006 et révisé en 2012 (279 p.) ${ }^{(12)}$, ainsi que l'ouvrage collectif dirigé par Hellmuth Lange, Nachhaltigkeit als radikaler Wandel. Die Quadratur des Kreises?, paru en 2008 $(341 \text { p. })^{(13)}$;

- enfin, toujours dans chacune des deux langues, deux publications récentes qui soulignent les questionnements renouvelés depuis la fin des années 2000, à travers l'exemple des énoncés de la décroissance/Postwachstum: en France, les deux tentatives de synthèse (engagée) proposées par Stéphane Lavignotte, La décroissance est-elle souhaitable?, en 2009 (137 p. $)^{(14)}$, et par Denis Bayon, Fabrice Flipo et François Schneider, La décroissance, publiée en 2010 et en format poche en 2012 (248 p. $)^{(15)}$; en Allemagne, deux ouvrages parus en 2016: celui de Marc Hieronimus, Der Schritt zur Seite: Postwachstum - Rückgang - Décroissance (328 p.) ${ }^{(16)}$, et un collectif (AK Postwachstum), Wachstum - Krise und Kritik (315 p.) ${ }^{(17)}$.

\section{Problématique}

Sur cette base, notre interrogation est double. D’une part, un grand nombre de définitions du «développement durable» ont été proposées en sciences sociales depuis quelque trente ans ${ }^{(18)}$. À la suite de nos précédents travaux, conduits sur un corpus de

8 Joseph Huber, Allgemeine Umweltsoziologie (2001), Wiesbaden, VS Verlag, 2011.

9 Lionel Charles, Hellmuth Lange, Bernard Kalaora et Florence Rudolf (dir.), Environnement et sciences sociales en France et en Allemagne, Paris, L'Harmattan, 2014.

10 Bertrand Zuindeau (dir.), Développement durable et territoire, Villeneuve d'Ascq, Presses universitaires du Septentrion, 2010.

11 Josiane Stoessel-Ritz, Maurice Blanc et Nicole Mathieu (dir.), Développement durable, communautés et sociétés. Dynamiques socio-anthropologiques, Bruxelles, PIE-Peter Lang, 2012.

12 Armin Grunwald et Jürgen Kopfmüller, Nachhaltigkeit (2006), Francfort, Campus Verlag, 2012.

13 Hellmuth Lange (éd.), Nachhaltigkeit als radikaler Wandel. Die Quadratur des Kreises?, Wiesbaden, VS Verlag, 2008.

14 Stéphane Lavignotte, La décroissance est-elle souhaitable?, Paris, Textuel, 2009.

15 Denis Bayon, Fabrice Flipo et François Schneider, La décroissance. Dix questions pour comprendre et débattre (2010), Paris, La Découverte, 2012.

16 Marc Hieronimus, Der Schritt zur Seite: Postwachstum-Rückgang-Décroissance, Norden, catware.net Verlag, 2016

17 AK Postwachstum (éd.), Wachstum - Krise und Kritik. Die Grenzen der kapitalistisch-industriellen Lebensweise, Francfort, Campus Verlag, 2016.

18 Les Handbooks internationaux en témoignent, y compris à mesure qu'une pluralité de disciplines s'intéressent à ces problématiques. Voir notamment Giles Atrinson, Simon Dietz et Eric 
revues scientifiques francophones et anglophones publiées de 2009 à 2014 ${ }^{(19)}$, l'on peut d'autant plus se demander dans quelle mesure ces définitions varient en fonction des traditions et des cadres nationaux. Ou, au contraire, se rejoignent-elles dans les deux contextes français et allemand, en fonction des circulations conceptuelles et pratiques internationales, de l'évolution des connaissances et des représentations des différents acteurs et institutions en la matière? À cela s'ajoutent de possibles problématiques de traduction, entre «soutenable» et «durable», par exemple, que nous n'aborderons pas ici, en nous concentrant sur la paire «développement durable»/Nachhaltige Entwicklung - «durabilité »/Nachhaltigkeit.

Nous considérons ce premier volet de deux façons : en voyant (1) en quoi le répertoire de la durabilité est «acté» en France et en Allemagne comme un objet pour les disciplines académiques, à travers l'exemple de manuels de sociologie de l'environnement; et (2) ce que désigne plus précisément ce registre en tant que tel au sein des analyses de sciences sociales dans les deux pays, à travers quelques ouvrages significatifs qui lui ont été consacrés.

D’autre part, les critiques adressées au paradigme du «développement durable» sont devenues nombreuses: continuité du primat d'un référentiel économique, permanence d'injustices sociales, voire production de nouvelles inégalités environnementales entre ceux qui auraient les moyens de bénéficier d'innovations techniques et les autres, références écologiques de compromis, à l'instar de la «croissance verte»/ grünes Wachstum, devenues outil de légitimation des décideurs publics et privés, dans un sens managérial et néolibéral, parfois éloigné de la perception des habitants, etc. ${ }^{(20)}$.

On peut comprendre en ce sens le fait, sur le plan international, que l'importante International Union for Conservation of Nature (IUCN) a pris ces dernières années quelque distance avec le terme de «développement durable». Pour preuve, son rapport Transition to Sustainability: Towards a Humane and Diverse World, en 2008, fait référence à la sustainability ou ecological sustainability, ce qui se veut différent du sustainable development, en désignant un état, ou une condition visant une stabilité, et non pas un processus maintenu de développement (économique) qui serait «soutenable».

Neumayer (éd.), Handbook of Sustainable Development, Cheltenham, Edward Elgar, 2007; ainsi que Michael Redclift et Delyse Springett (éd.), Routledge International Handbook of Sustainable Development, Londres/New York, Routledge, 2015.

19 Si l'on cible la «ville durable», ces répertoires de la durabilité sont apparus caractérisés par de nombreux traits communs, en termes de contenus et de procédures: Philippe Hamman, «Pollution atmosphérique, climat et société: quelle place aujourd'hui dans la "ville durable” ?", Pollution atmosphérique. Climat, santé, société, 233 (2017), en ligne: lodel.irevues.inist.fr/pollution-atmospherique/ index.php?id=5216; Hamman/Anquetin/Monicolle, «Contemporary Meanings » et «Du "développement durable"» (note 4$)$.

20 Ainsi que l'ont montré les recherches sur la «ville durable» en Allemagne comme en France, par exemple: Tim Freytag, Stefan Gössling et Samuel Mössner, «Living the Green City: Freiburg's Solarsiedlung between Narratives and Practices of Sustainable Urban Development ", Local Environment, 19/6 (2014), p. 644-659; Philippe HAMmAN, «Ökonomische und soziokulturelle Ungleichheiten in Bezug auf einen ökologischen Wandel: Die Energiewende am Beispiel von Straßburg», Revue d'Allemagne et des pays de langue allemande, 49/1 (2017), p. 223-242; Philippe Hamman, "Local Governance of Energy Transition: Sustainability, Transactions and Social Ties. A Case Study in North East France», International Journal of Sustainable Development and World Ecology, 26 (2019), en ligne: doi.org/10.10 80/13504509.2018.1471012; Jérôme Boissonade (dir.), La ville durable controversée, Paris, Petra, 2015. 
Des évolutions multiniveaux s'opèrent aujourd'hui entre des échelles élargies/ mondiales et d'autres davantage territorialisées autour de cette notion duale, à la fois analytique et ancrée dans la société; ceci se repère tant en France qu'en Allemagne. Plusieurs ouvrages publiés depuis la deuxième moitié des années 2000 l'illustrent. Il en est ainsi, côté allemand, du manuel Nachhaltigkeit d'Armin Grunwald et Jürgen Kopfmüller, où la distinction est posée comme suit: "Während nachhaltige Entwicklung einen Prozess gesellschaftlicher Veränderung bezeichnet, beschreibt Nachhaltigkeit das Ende eines solchen Entwicklungsprozesses» (édition de 2006, p. 1). Le distinguo est repris comme tel par Kristina Schaaff dans la présentation de son texte éponyme Nachhaltigkeit publié en 2007, qui ajoute: «Während das Drei-SäulenModell ${ }^{(21)}$ der Nachhaltigkeit zunehmend an Bedeutung verliert, findet das integrative Nachhaltigkeitsmodell [...] wachsenden Anklang» ${ }^{(22)}$. Côté français, notre analyse bibliométrique a montré que l'association entre développement et durable n'apparaît pas/plus systématique dans la littérature à la fin des années $2000^{(23)}$. On peut aussi citer la parution en 2014 de l'ouvrage de Yannick Brun-Picard, intitulé de façon significative Plus loin que le développement durable: la durabilité, où l'auteur énonce que cette dernière "va bien plus loin que la notion de développement durable», parlant d'une "conscientisation et d'une responsabilisation par l'intermédiaire de laquelle la surface terrestre est acceptée comme un partenaire vivant », c'est-à-dire que «nos sociétés sont impliquées et indissociables des conséquences des actions entreprises » ${ }^{(24)}$.

D’aucuns se positionnent plus encore en rupture, à l'instar de Dennis Meadows, qui écrit en 2013: «Il est trop tard pour le développement durable » ${ }^{(25)}$, ce qui s'accompagne d'un retour du/au paradigme de la «décroissance». Ce dernier - tout comme la durabilité, on l'a dit - peut se lire de diverses manières selon le contexte dans lequel on se situe:

- la décroissance peut être vue, à l'heure actuelle, comme une alternative au «développement durable», en particulier à propos de la controverse de la compatibilité ou non entre durabilité et croissance ${ }^{(26)}$;

- mais également comme un répertoire d'écologie politique antérieur à la formulation des énoncés contemporains de la durabilité, depuis les années 1970, si l’on pense notamment aux travaux de Nicholas Georgescu-Roegen et à la loi de l'entropie ${ }^{(27)}$, ou

21 C'est-à-dire la quête permanente et toujours relative d'équilibre entre les trois piliers économique, écologique et social de la durabilité.

22 Grunwald/Kopfmüller, Nachhaltigkeit (note 12); Kristina SchaAfF, Nachhaltigkeit, Munich, Grin Verlag, 2007.

23 Philippe Hamman, «Definitions and Redefinitions of Urban Sustainability. A Bibliometric Approach», Urban Environment, 11 (2017), en ligne: eue.revues.org/1540.

24 Yannick Brun-Picard, Plus loin que le développement durable: la durabilité, Paris, L'Harmattan, 2014, p. 226.

25 Dennis Meadows, «Il est trop tard pour le développement durable», in: Agnès Sinaï (dir.), Penser la décroissance. Politiques de l'Anthropocène, Paris, Presses de Sciences Po, 2013, p. 195-210.

26 Sur ce débat central de la durabilité, voir Peter A. Victor et Brett Dolter (éd.), Handbook on Growth and Sustainability, Cheltenham, Edward Elgar, 2017.

27 Nicholas Georgescu-Roegen, La décroissance. Entropie, écologie, économie (1979), Paris, Sang de la Terre, 2006. 
ceux d'Ivan Illich et la notion de convivialité( ${ }^{28)}$, qui fait à nouveau l'objet de débats conséquents depuis les années 2000 dans les mouvements intellectuels et militants en France et en Allemagne - des publications s'en font l'écho de part et d'autre ${ }^{(29)}$;

- ou encore, bien avant, comme une critique du progrès et de la modernité qui a émergé en Europe dans des mouvements politiques et philosophiques de droite comme de gauche ${ }^{(30)}$.

À partir du registre de la «décroissance», ce deuxième volet consiste ainsi à poser la problématique de l'alternative en tant que telle, en un temps parfois qualifié de «post-politique» ${ }^{(31)}$, où le débat public a souvent fait l'objet d'élisions lorsqu'est mis en avant l'avenir de la planète, et cela alors que la durabilité est une question proprement politique $^{(32)}$. La décision du président américain Donald Trump en juin 2017 de quitter l'Accord de Paris sur le climat conclu en décembre 2015 le traduit nettement: la prévalence d'intérêts économiques assumée par rapport aux menaces du réchauffement climatique rappelle qu'il n'existe pas de légitimité «en surplomb».

\section{Trois indicateurs en perspective comparée}

Une reconnaissance de l'objet «durabilité » en sciences sociales:

l'exemple des manuels de sociologie de l'environnement

L'analyse des quatre manuels de sociologie de l'environnement considérés permet de dégager cinq traits notables qui se retrouvent dans les deux pays.

1. Le premier constat est celui de la présence désormais incontournable de l'énoncé et de l'objet «durabilité» dans ces manuels. Le terme ne va pas de soi pour une discipline qui étudie les relations nature/société et s'est, à ce titre, consacrée à interroger les notions de nature, d'environnement ou encore d'écologie ${ }^{(33)}$. Désormais, chacun de ces manuels intègre un questionnement de la durabilité énoncé en tant que tel dans la structure de l'ouvrage: deux chapitres y sont dédiés dans le Manuel de sociologie de l'environnement, une section explicite dans Environnement et société (et plusieurs autres sous-sections en traitent de facto aussi), une section est également affichée dans Allgemeine Umweltsoziologie (et plusieurs sous-sections en relation), ainsi que l'une des cinq parties du Handbuch Umweltsoziologie et des chapitres d'autres parties.

28 Ivan Illich, La convivialité (1973), Paris, Points-Seuil, 2014.

29 Voir Alain Caillé, Marc Humbert, Serge Latouche et Patrick Viveret, De la convivialité. Dialogues sur la société conviviale à venir, Paris, La Découverte, 2011; Frank Adloff et Volker M. Heins (éd.), Konvivialismus. Eine Debatte, Bielefeld, Transcript Verlag, 2015.

30 Pour une mise en perspective, voir Dominique Bourg et Augustin Fragnière, La pensée écologique. Une anthologie, Paris, Presses universitaires de France, $2014,2^{\mathrm{e}}$ partie.

31 Slavoj ŽıžEk, The Ticklish Subject: The Absent Centre of Political Ontology, Londres, Verso, 1999 (traduction française: Le sujet qui fâche. Le centre absent de l'ontologie politique, Paris, Flammarion, 2007).

32 Henrik Ernstson et Erik Swyngedouw (éd.), Urban Political Ecology in the Anthropo-obscene. Political Interruptions and Possibilities, Londres/New York, Routledge, 2017; ainsi que Hamman/ Anquetin/Monicolle, "Contemporary Meanings », et «Du “développement durable” " (note 4).

33 Pour un point, voir notre contribution: "Les relations entre ruralité, nature et environnement en sciences sociales: mises en perspective et renouvellement», in: Philippe Hamman (dir.), Ruralité, nature et environnement. Entre savoirs et imaginaires, Toulouse, Érès, 2017, p. 7-58. 
Au-delà, on retrouve trois modes d'entrée, signe d'une porosité des contextes nationaux en regard des développements multiniveaux de la durabilité.

2. Tout d'abord, à chaque fois est étudiée la genèse et la consécration internationale du registre de la durabilité, ainsi positionné et légitimé à l'échelle globale. Dans le Manuel de sociologie de l'environnement, un chapitre intitulé «De l'écodéveloppement au développement durable» y est pleinement consacré et s'attache à dépeindre un cheminement en quatre temps: les crises du développement qui sont objet de débats dans les années 1970 - sous l'angle des limites de la croissance des activités économiques, mais aussi de la croissance démographique - constituent le contexte de l'émergence de l'«écodéveloppement», notamment forgé par Ignacy Sachs ${ }^{(34)}$, «comme le double refus de deux radicalismes: l'économisme et l'écologisme. [...] Le premier met en cause un système économique qui érige la production comme fin en soi. [...] Le second évince l'homme d'une autre manière, [...] la conservation de la nature étant alors la finalité suprême. [...] L'écodéveloppement se veut donc une voie médiane» (p. 246247). Puis il est montré en quoi, à partir du début des années 1980, cette notion cède progressivement la place à celle de développement durable. Les fils conducteurs sont le balancement "substitutions de notions, permanence des idées» (p. 251) et la polysémie du développement durable, laquelle favorise d'abord la convergence, puis vient à marquer des antagonismes, d'où "plus récemment la montée en force du courant de la décroissance» (p. 255). De même, si Environnement et société situe ses analyses surtout dans le cadre français, en examinant la montée en puissance du développement durable en lien avec «la prise en charge publique du champ de l'environnement» (objet du chapitre III), la mise en perspective socio-historique des évolutions du ministère de l'Environnement est l'occasion d'étudier le processus de «la normalisation du développement durable» et de revenir ainsi sur «le cheminement de la notion de développement durable [qui] est aussi celui de la formulation politique des enjeux environnementaux", où l'on retrouve une mise en perspective internationale à partir des années 1970 et passant par la théorie de l'écodéveloppement (p. 99-101).

Le constat dressé et le cheminement restitué sont similaires dans les deux manuels allemands. Ainsi, la section intitulée «Nachhaltige Entwicklung» dans Allgemeine Umweltsoziologie dégage-t-elle là aussi «das Leitbild der Nachhaltigen Entwicklung» (p. 155 sq.) en se situant à l'échelle internationale et en étudiant comme cadres structurants le rapport Brundtland de 1987 et le sommet de la Terre de Rio de 1992, sans oublier de revenir aux années 1970 et à l'écodéveloppement (p. 158-159). Qui plus est, un autre chapitre étudie également cette assise internationale de la problématique de la durabilité, en la liant à celle de la gouvernance mondiale: «Internationale Umweltregime. Global Environmental Governance» (p. 242-254). Cette même perspective globale se retrouve enfin dans le Handbuch Umweltsoziologie. Un chapitre traite expressément de la «Soziologie globaler Umwelt- und Nachhaltigkeitspolitik», avec des développements là encore scandés à partir des institutions et conférences internationales (p. 628-650). Deux autres chapitres, situés en fin de volume et ouvrant des perspectives, font aussi le lien avec la question "globale» du changement climatique,

34 Voir en particulier Ignacy SAchs, Stratégies de l'éco-développement, Paris, Éditions Économie et Humanisme/Éditions ouvrières, 1980. 
qui prend une place de plus en plus forte en matière de durabilité: «Die Politisierung des globalen Klimawandels und die Konstitution des transnationalen Klimaregimes» et «Klimawandel und globale Umweltveränderungen» (p. 671-720).

Derrière ces similarités, une spécificité est toutefois remarquable dans les ouvrages allemands. À la différence des écrits francophones (ou anglo-saxons), ils rapportent couramment le registre de la durabilité à une pré-histoire de long terme à travers le «Waldbewirtschaftungsprinzip» énoncé en 1713 par Carl von Carlowitz. Ceci se retrouve par exemple dans le manuel Nachhaltigkeit d'Armin Grunwald et Jürgen Kopfmüller, lorsque les auteurs déclinent leur chapitre socio-historique: avant de restituer les débats internationaux («Internationale Debatten über Umwelt und Entwicklung»), une première section («Ursprünge des Leitbildes nachhaltiger Entwicklung», p. 18-20) attribue au concept de durabilité des origines plus lointaines dans les études forestières:

«Der Begriff "Nachhaltigkeit” tauchte erstmals Anfang des 18. Jahrhunderts in der Forstwirtschaft unter der Zielsetzung auf, ökonomische Erwägungen mit dem Faktor Natur in Einklang zu bringen. Vielfach wird die Abhandlung Sylvicultura Oeconomica des sächsischen Oberberghauptmanns von Carlowitz aus dem Jahr 1713 als erstmalige Erwähnung genannt. [...] Es sollte pro Jahr nicht mehr Holz geschlagen werden als nachwächst. Dieses (ressourcenökonomische) Prinzip [...] wurde ein Vorbild für spätere Nachhaltigkeitsüberlegungen» (p. 18-19).

Cette filiation singulière est largement partagée dans les analyses germaniques et se repère de manuel en manuel, à l'instar de celui d'Iris Pufé, Nachhaltigkeit. Là aussi, le chapitre «Geschichte der Nachhaltigkeit» débute par une section dédiée à Carlowitz et à sa postérité, affiliant la durabilité à un principe initial de gestion des ressources naturelles, et en particulier des forêts («Seinen Ursprung hat der Begriff [Nachhaltigkeit] in der Forstwirtschaft», pose l'auteure), avant de citer également les prémices de l'écologie à travers les écrits de Carl von Linné (Systema Naturae, 1735) ${ }^{(35)}$.

3. Se dégage ensuite le lien entre développement durable et innovation ou modernisation, et notamment la connexion avec les théories de la modernisation écologique, dont l'Allemagne a été le berceau avec les travaux de Joseph Huber (avant une extension européenne puis internationale via l'école néerlandaise autour notamment d'Arthur P. J. Mol et Gert Spaargaren ${ }^{(36)}$ ), et qui se sont également diffusées en France.

Sans surprise, les deux manuels allemands de sociologie de l'environnement retenus y consacrent des développements substantiels. Joseph Huber lui-même a rédigé le manuel Allgemeine Umweltsoziologie; il y synthétise la thèse de la modernisation écologique («ökologische Modernisierung»), qui se fonde sur l'hypothèse d'une possible compatibilité entre économie et écologie - à l'opposé du postulat des tenants de la décroissance:

«Die Strategie der ökologischen Modernisierung geht davon aus, dass Ökonomie und Ökologie, Industrie und Natur, nicht zwangslaüfig Gegensätze zu sein brauchen. Sie lassen sich so weit miteinander in Einklang bringen, wie es gelingt, die Steigerung der Umweltproduktivität - als Ressourcen-, Energie- und Senkenproduktivität -, zu einer ebensolchen Wohlstandsquelle zu machen wie die bisherige Arbeitsproduktivität» (p. 143). 
Dans son analyse en termes de métabolisme industriel, Joseph Huber met l'accent sur les innovations technologiques («technologische Umweltinnovationen»), tant dans le domaine de l'énergie que des flux de matières, comme clef de la durabilité: «Bleibt als realistischer Ausweg: innovativer Strukturwandel, der die ökologische Tragekapazität für die Menschheit einmal mehr erhöht»(p. 171). Des jalons sont posés dans le chapitre significativement intitulé «Ökologische Diskurse, Leitbilder und Strategien. Vom Nullwachstum zur ökologischen Modernisierung» (p. 134-182), qui intègre la présentation du développement durable entre les débats sur la croissance et ceux sur la modernisation écologique. Puis l'auteur développe ce paradigme autour de deux grandes entrées: «Umweltmanagement. Ökologisch wirtschaften und produzieren » et "Ökoindustrielle Wirtschaftsentwicklung» (p. 255 sq. et 276 sq.). De façon proche, le Handbuch Umweltsoziologie accueille un chapitre signé de Joseph Huber, qui déplie le même fil directeur: "Ökologische Modernisierung und Umweltinnovation» (p. 279 sq.), ainsi qu'un autre sous la plume des promoteurs néerlandais de la modernisation écologique, Arthur P. J. Mol et Gert Spaargaren : «Zur Umweltsoziologie der Netzwerke und Flows» (p. 140 sq.), où ils s'intéressent en particulier aux flux de matières. Plus largement, l'ouvrage consacre explicitement l'une de ses grandes parties aux rapports entre "Modernisierung, Innovation und Nachhaltigkeit», dépliés en sept chapitres qui dégagent transversalement une lecture d'abord experte et technique de la durabilité, vue comme accommodement par l'innovation, notamment, pour reprendre des têtes de chapitres, en matière de services écosystémiques, de mobilité, d'énergie et de déchets (p. 277-442).

Cette diffusion des théories de la modernisation écologique ressort également en France. Cela apparaît certes à un degré moindre dans le Manuel de sociologie de l'environnement, qui n'y consacre pas de chapitre ou section affichés en propre. C'est là peutêtre un signe de ce que la notion n'a pas son origine dans le contexte francophone, au contraire du cadre allemand qui la promeut. Pour autant, elle est bien mobilisée au fil des textes. Et sa reconnaissance est plus nette dans Environnement et société, où une section aborde la «modernisation écologique» comme «l'institutionnalisation d'une gestion économique de l'environnement» (p. 136-142). Les auteures explicitent «le développement d'un marché [économique] fondé sur la valeur environnementale de ses productions, que ce soit dans le domaine de l'agroalimentaire, des technologies propres, des entreprises de traitements, etc., permettant la création de "niches économiques" allant des "écoproduits" à "l'industrie écologique" "; et d'ajouter: «L'intégration économique des problèmes de pollution a ainsi été un moteur de la croissance, permettant non seulement de renouveler le process mais aussi d'assurer le développement d'un secteur des "biens et services environnementaux" " (p. 136-138). Les exemples donnés au niveau des déchets et flux de matières rejoignent également la synthèse réalisée dans les manuels allemands.

On relève deux nuances par rapport aux présentations allemandes. D’une part, ce ne sont pas les travaux initiaux de Joseph Huber qui sont cités et exposés, mais les élaborations d'Arthur P. J. Mol et Gert Spaargaren. On lit ainsi dans Environnement et société:

«Ce processus défini dans la littérature anglo-saxonne sous le terme d'“ecological modernization" est présenté par les auteurs de ce courant en sciences sociales comme une "vision optimiste" et "réformatrice" des relations entre protection de l'environnement et croissance 
$\left(\right.$ Mol et al. 2009) ${ }^{(37)}$. Le processus d'industrialisation par l'innovation technologique doit être soutenu par une réforme des institutions politiques qui permet une participation croissante des acteurs économiques et sociaux à la modernité écologique» (p. 139).

Le constat est corroboré à la lecture du Manuel de sociologie de l'environnement, où l'on dénombre en bibliographie pas moins de cinq références aux publications d'Arthur P. J. Mol et ses collègues néerlandais, et seule une référence à Joseph Huber, cité dans le chapitre consacré à la sociologie allemande de l'environnement pour la réception de son manuel Allgemeine Umweltsoziologie de façon générale. On voit ici de facto que les auteurs néerlandais ont été centraux dans la diffusion internationale - et non pas directement d'Allemagne en France - de la notion. Joseph Huber le reconnaît du reste dans Allgemeine Umweltsoziologie, dès le début de la section portant sur la modernisation écologique: «Durch Rezeption und weitere Ausarbeitung bei den holländischen Umweltsoziologen Mol und Spaargaren hat der Ansatz erst europaweit, dann auch weltweit Aufnahme gefunden» (p. 143).

D'autre part, on note un regard plus directement critique sur la modernisation écologique que dans les manuels allemands, ce qui peut s'expliquer par la position pionnière de Joseph Huber en la matière en Allemagne. En France, dans Environnement et société, la présentation de la notion est suivie d'une mise à distance au titre de la problématique des «inégalités environnementales»: "L'ajustement de ce mode de développement se fait par le creusement d'inégalités quant à l'accès aux ressources naturelles et à l'exposition aux pollutions» (p. 141). Ceci renvoie aussi plus largement à la place de la sociologie critique française, qu'incarne par exemple Jean-Louis Fabiani lorsqu'il met en garde contre les soubassements de la modernisation écologique:

«La modernisation écologique peut être considérée comme le point d'aboutissement de la dissociation entre, d'un côté, une forme activiste de l'écologie, laquelle, avec des degrés de radicalité divers, a constitué la question environnementale comme problème public, et a donc été le vecteur principal de son institutionnalisation, et, d'un autre côté, une forme experte, qui s'est efforcée de dépolitiser l'objet et de le rendre compatible avec les modes de gestion techno-bureaucratiques susceptibles de légitimer son expertise » ${ }^{(38)}$.

Ce rôle croissant de l'expertise fait d'ailleurs l'objet d'un chapitre dans Environnement et société, ciblant «le recours à l'expertise comme nouveau mode de gouvernement de l'incertitude» (p. 179 sq.). Les enjeux des inégalités environnementales ne sont pas ignorés côté allemand, mais, par exemple, le chapitre qui en traite dans le Handbuch Umweltsoziologie («Umweltgerechtigkeit», p. 464-484) est présenté de façon bien distincte de celui dédié à la modernisation écologique («Ökologische Modernisierung und Umweltinnovation», p. 279-302), sans discussion croisée entre les concepts et/ou auteurs, même si toutes les pièces du panorama sont bel et bien fournies.

4. Un dernier trait significatif tient aux enjeux d'implication et de pratiques citoyennes rapportés aux démarches de durabilité. Cette problématique est nettement posée dans Environnement et société, sous l'angle de «l'éducation à l'environnement, entre critique et adaptation», que l'on sait largement reprise sous le registre d'«éducation au

37 Référence à Mol/SPAARgAREN, The Ecological Modernisation Reader (note 36).

38 Jean-Louis FABiAni, «Rural, environnement, sociologie», in: P. Hamman, Ruralité, nature et environnement (note 33), p. 111-132, ici p. 114. 
développement durable» ${ }^{(39)}$, avec en arrière-plan le fait que «la diffusion contemporaine de la notion d'écocitoyenneté renvoie explicitement aux valeurs de la morale écologique, dans le sens où elle renomme l'acteur social comme étant en relation avec un écosystème. Celui-ci participe par agrégation en tant qu'individu à une œuvre collective et peut accéder ainsi au statut de citoyen » (p. 241). Chantal Aspe et Marie Jacqué montrent que ce postulat n'est pas neutre; il emporte une «normalisation de l'engagement écocitoyen", où le discours des «bonnes pratiques» environnementales peut devenir "aliénant». L'écocitoyenneté s'appuie en effet «sur un traitement économique et technique des objets environnementaux» et opère un transfert de responsabilité du collectif vers l'individu, invisibilisant le poids des structures socio-économiques et autres rapports de force (p. 241-253). Sans que l'on y trouve cette fois de développements spécifiques, l'association entre (appel à la) durabilité dans l'action publique et participation citoyenne est aussi relevée dans le Manuel de sociologie de l'environnement, dans le chapitre portant sur la gouvernance de la durabilité, qui explicite les modes de cadrage (procédures, limitation aux échelles de proximité...) de ce «faire participer» (p. 271-272).

Côté allemand, le manuel Allgemeine Umweltsoziologie consacre également son dernier chapitre à l'écocitoyenneté: «Umweltbewusstes Verbraucherverhalten, ökologische Haushaltsführung» (p. 295-315), se situant ainsi à l'échelle des pratiques individuelles - après avoir préalablement examiné le registre du développement durable sous l'angle des stratégies d'efficience («Effizienzstrategie», rattachée à la conférence de Rio) et de la modernisation écologique (en termes de métabolisme et d'éco-innovations technologiques). Tout comme le manuel français Environnement et société, on repère une prise de distance, mais la posture diffère. Là où la sociologie critique française relève une possible «aliénation" des individus ou normalisation imposée de leurs pratiques par les décideurs au nom de la durabilité comme répertoire de légitimation (les fameuses "bonnes pratiques») ${ }^{(40)}$, Joseph Huber dénonce quant à lui les marqueurs d'un «fondamentalisme écologique» (p. 304). Le point commun est de rapporter la durabilité à un cadre de légitimation pour des impositions, jusque, par exemple, dans la façon dont les questions adressées aux citoyens sont formulées dans les enquêtes sur l'écoconsommation:

«Die Items kreisen darum, (a) mit weniger zufrieden zu sein und (b) mehr dafür zu bezahlen. Die Items sind unter diesem Aspekt in dreifacher Hinsicht problematisch - erstens im Hinblick auf die Validität der Items, zweitens hinsichtlich ihrer ökologischen Relevanz, drittens und nicht zuletzt unter ideologiekritischen Aspekten» (p. 302).

Corrélativement, une section invite à resituer ces enjeux par rapport aux variables et clivages socio-démographiques et économiques, c'est-à-dire à les replacer dans l'épaisseur du social, rejoignant de la sorte Environnement et société:

39 Voir notamment Philippe Hamman et Christine Blanc, Sociologie du développement durable urbain, Bruxelles, PIE-Peter Lang, 2009.

40 On lit ainsi: «L'écocitoyenneté focalise un désir d'engagement pour l'environnement, qui, en s'exprimant dans des cadres gestionnaires, devient aliénant pour l'individu, lequel est dessaisi de la portée politique de son acte et détourné de l’objectif de son engagement. Par cet engagement, il participe paradoxalement aux formes dominantes d'exploitation des ressources naturelles et au maintien d'un mode de vie axé sur la consommation, ce qu'il est censé combattre», AsPe/JACQUÉ, Environnement et société (note 6), p. 251. 
«Soziologisch gesehen ist es selbstverständlich, Umweltbewusstsein und Umweltverhalten auf demographische Merkmale wie Alter und Geschlecht zu beziehen, ebenso auf Merkmale der sozialen Lage wie Schicht- und Milieuzugehörigkeit. Davon ausgehend lag es nahe, umweltbewusstes Verbraucherverhalten auch auf Lebensstile zu beziehen» (p. 305).

Quant au Handbuch Umweltsoziologie, il déploie - tel un pendant au chapitre consacré à la gouvernance de la durabilité dans le Manuel de sociologie de l'environnement qui a aussi évoqué la dimension participative - un chapitre consacré à «Partizipation und neue Formen der Governance» (p. 485-502), dont la ligne directrice énonce:

«Ein Hauptmotiv für diese Entwicklung liegt in der gewachsenen Komplexität moderner Umwelt- und Nachhaltigkeitsprobleme begründet, der man mit flexibleren, anpassungsfähigeren Formen gesellschaftlicher Entscheidungsfindung begegnen möchte, die zugleich derartige Entscheidungen auf eine breitere gesellschaftliche Basis stellen» (p. 485).

Il s'ensuit des enjeux de cadrage (niveaux d'association du citoyen) et d'objectifs divers, explicités à partir d'une triade émancipation/légitimation/effectivité:

«Emanzipation beschreibt die Befreiung aus Zuständen der Abhängigkeit bzw. Ungleichheit und eine (Wieder-)Gewinnung von Selbstbestimmung, wie sie für demokratische Gesellschaften prägend ist. [...] Partizipation im Sinne einer Teilhabe an kollektiven Entscheidungen bildet damit ein zentrales emanzipatorisches Element und ist als Kritik an herrschenden gesellschaftlichen Zuständen vor allem im marxistischen und neo-marxistischen Schriftum verankert. [...] Ein zweiter wichtiger Diskurs im Zusammenhang mit Partizipation bezieht sich auf die demokratischen Qualitäten und damit die Legitimität von Entscheidungsprozessen. [...] Der heute dominante Diskurs ist jedoch der einer zivilgesellschaftlichen Einbindung "von oben". Was wie ein Widerspruch in sich klingt, meint die von Seiten der Entscheidungsträger angestoßenen Möglichkeiten zur Beteiligung und Mitwirkung von üblicherweise nicht entscheidungsbefugten individuellen Akteuren, Institutionen, Organisationen, etc.» (p. 489-492).

5. Enfin, d'un point de vue synthétique, la lecture des quatre manuels considérés nous apprend qu'en France comme en Allemagne, les analyses consacrées au développement durable sous l'angle de la sociologie de l'environnement ne s'arrêtent pas tant à la conciliation du triptyque économie-écologie-social, c'est-à-dire le modèle dit des trois piliers de la durabilité, dans la filiation du rapport Brundtland de 1987. Ils soulignent plutôt deux axes en tension: le rapport à l'économie et à la croissance, et le rapport à la nature et à l'environnement, c'est-à-dire technocentrisme vs. écocentrisme. En ce sens, il en va dans la littérature retranscrite dans les manuels, tantôt d'une perspective intégrative et réformiste du processus de mise en durabilité - incarnée notamment par la «modernisation écologique», qui apparaît avoir pris le dessus en Allemagne dans les analyses de la durabilité, au moins un temps - , tantôt d'une lecture davantage sociale et critique - sachant qu'à présent la question de la justice environnementale et sociale est également de plus en plus investie dans les deux cadres nationaux ${ }^{(41)}$. Ce dernier aspect traduit aussi la dimension de la reconnaissance/réhabilitation des savoirs locaux sur la nature, à partir de la question environnementale portée par le développement durable. Ceci rappelle en filigrane que l'écodéveloppement, au sens

41 Voir les analyses convergentes de Karl-Werner Brand, "Sustainability Transition". La durabilité, défi social et politique», in: Charles/Lange/Kalaora/Rudolf, Environnement et sciences sociales (note 9), p. 117-146. 
d'Ignacy Sachs, relevé dans les différents manuels suggère une pluralité des voies de développement, c'est-à-dire de ne pas opposer artificialité et naturalité d'un écosystème, et de considérer les pratiques locales comme un point de départ de la résolution des problèmes techniques ${ }^{(42)}$.

\section{Quelle durabilité? Entre contenus et procédures}

Ces premiers constats complexifient déjà l'appréhension de la durabilité par-delà la seule question du compromis, toujours partiel et à recommencer de séquence en séquence, entre les trois pans économique, environnemental et social. Pour aller plus loin, nous focalisons sur deux ouvrages de référence, en France et en Allemagne, spécifiquement consacrés à l'analyse du développement durable, et publiés au cours ou à l'issue des années 2000 , qui ont marqué le succès de la diffusion de la notion. Nous ne revenons pas sur les traits précédemment dégagés, qui sont à nouveau attestés. Par exemple, on retrouve à chaque fois le rappel des dynamiques de genèse et de consécration de la notion, largement rapportées à la scène internationale. De façon révélatrice, le manuel devenu classique d'Armin Grunwald et Jürgen Kopfmüller, Nachhaltigkeit, s'ouvre par un premier chapitre dépeignant cette histoire: «Entstehungsgeschichte und wesentliche Meilensteine» (p. 18 sq.). Les auteurs y présentent les débats internationaux sur l'environnement et la croissance et des moments consacrés, comme la commission Brundtland, le sommet de Rio et les Objectifs du millénaire pour le développement, dans le cadre des Nations unies.

Dans cette littérature spécialisée, en France et en Allemagne, on note de façon centrale les rapports, toujours en train de se faire, entre contenus et procédures de la durabilité, tant en termes de savoirs que de pratiques. Cette ligne directrice présente de réelles similitudes dans les deux pays et se caractérise à partir de cinq entrées et modes d'analyse principaux, dont rendent compte les manuels.

\section{Durabilité faible et forte}

En interaction avec le trinôme économie/écologie/social, Bertrand Zuindeau positionne cet enjeu dès l'introduction de Développement durable et territoire: «Entre les approches "plutôt économiques" ou, au contraire, "plutôt écologiques" du DD [développement durable], entre ce que les théoriciens du DD ont convenu d'appeler, d'un côté, la "durabilité faible" et, de l'autre, la "durabilité forte", il n’y a pas que des nuances, tant s'en faut! » ${ }^{(43)}$. L'auteur explicite: «Une conception est dite de "durabilité faible" si elle estime que les diverses composantes du capital (capital matériel, capital humain, capital naturel) sont substituables. À l'inverse, les approches de type écologique retiennent plutôt l'idée que cette substitution n'est pas possible». En même temps, il invite à considérer que «la prise en compte des trois piliers du DD (l'économique, le

42 Voir Florence Pinton, «De la mise en valeur coloniale au développement durable: le statut des savoirs locaux sur la nature», in: Charles/LAnge/Kalaora/Rudolf, Environnement et sciences sociales (note 9), p. 343-367.

43 Sachant que, sous d'autres aspects, l'on trouve aussi une lecture duale en littérature, entre "écologie superficielle» et "écologie profonde», par exemple. Voir notamment Hicham-Stéphane AfEISSA, "Ecosophy: "How deep is your ecology"?», in: Aurélie Choné, Isabelle Hajek et Philippe HamMAN (dir.), Rethinking Nature, Challenging Disciplinary Boundaries, Londres/New York, Routledge, 2017, p. 27-37. 
social, l'environnemental) ne doit pas conduire à un simple collage, mais doit donner lieu à une véritable intégration, amenant à redéfinir les objectifs du développement eux-mêmes» (p. 14-15). Cette attention se confirme au fil des chapitres, à l'instar de celui sur «les antécédents conceptuels du développement soutenable» (p. 25 sq.) ou de la réflexion sur «équité territoriale et développement durable» (p. 97-107).

La position exprimée dans le manuel Nachhaltigkeit est comparable, pointant les débats «Starke oder schwache Nachhaltigkeit? » et « Nachhaltigkeit und Wirtschaftswachstum» comme structurants de la durabilité (chap. 4), et non uniquement le trinôme économie/ écologie/social, parfois présenté comme le «"triangle” magique de la durabilité» («das magische "Dreieck" der Nachhaltigkeit», p. 58). Ce même arrière-plan de durabilité forte ou faible est également présent dans Nachhaltigkeit als radikaler Wandel. L'un des trois défis centraux dégagés par Hellmuth Lange, afin de dépasser «un correctif purement esthétique de la dynamique de développement existante» («eine bloß kosmetische Korrektur der gegebenen Entwicklungsdynamik», citant ici Carsten Wiemeyer ${ }^{(44)}$ ), est celui des objectifs («Ziele») à formuler par rapport à cette double grille:

«Einerseits zur Stabilisierung der Ressourcenbasis und der tragenden ökosystemaren Zusammenhänge der Erde und andererseits in einer Verteilung der Kosten, des Nachhaltigkeitswandels, die in der gesellschaftlichen Breite als einigermaßen akzeptabler neuer Angemessenheits- und Gerechtigkeitsrahmen verstanden wird - nicht zuletzt im NordSüd-Verhältnis» (p. 36).

Il devient alors de plus en plus fréquent d'identifier la démocratie comme un quatrième pan de la durabilité. Armin Grunwald et Jürgen Kopfmüller soulignent cette dimension politico-institutionnelle ("politisch-institutionnelle Dimension»), et en particulier l'importance de la démocratie et de la participation comme modes de légitimation des processus décisionnels (p. 40). Dans l'ouvrage collectif Développement durable, communautés et sociétés, deux chapitres s'attachent pleinement à cette question du «Développement durable démocratique (DDD)». Notablement, dans son texte conclusif, le sociologue André Petitat, alors président de l'Association internationale des sociologues de langue française, en fait même le troisième pilier du DD, en lieu et place du social, écrivant: "La configuration normative du développement durable (DDD disent certains) [...] se trouve au carrefour de plusieurs dimensions, dont les trois principales sont l'économie (Développement), l'écologie (Durable) et la politique (Démocratie)» (p. 221). Plus largement, l'ouvrage montre que la mise en œuvre de politiques locales «durables» passe par des modes collectifs de délibération et de transaction. À ce titre, la durabilité relève d'une mise en rapport d'impératifs a priori éloignés (durabilité forte/faible, etc.), qui rejoint le répertoire également protéiforme de la gouvernance locale: "gouvernance démocratique et développement durable» sont examinés de pair par Maurice Blanc, dépliant "gouvernance et partenariat ", "gouvernance multi-niveaux» et "gouvernance intersectorielle» (p. 40-43).

2. Les configurations d'acteurs

Justement en lien avec la problématique de la gouvernance, une seconde caractéristique transversale porte sur les configurations d'acteurs et leur analyse dans le

44 Carsten Wiemeyer, "Sustainable Development» und die lokale Agenda 21 : Ein neues Arrangement auf dem Weg zur Zukunftsfähigkeit?, Marburg, Tectum, 2002, p. 23. 
cadre de démarches et projets en développement durable. L'ensemble de la partie V de Développement durable et territoire déplie les «Démarches d'acteurs » (p. 409-501) en termes de projets d'aménagement, de mobilisations collectives, d'Agendas 21 ou de Plans Climat locaux, d'écologie industrielle, de «bonnes pratiques» ou encore de "gouvernance». Transversalement, sont égrenés les aspects suivants: «information, formation, sensibilisation, appropriation, incitation, contrainte, conflits, coopération, etc.» (suivant les termes de Bertrand Zuindeau dans son introduction, p. 20-21). L'étude des jeux d'acteurs se retrouve au fil des pages de Développement durable, communautés et sociétés, à la fois dans le domaine de l'agriculture et celui de la ville, qui scandent les parties 2 et 3 du livre, précédées d'un chapitre rédigé par Marc Mormont, qui s’interroge sur «quels publics pour le développement durable?» (p. 53-65).

L'attention aux configurations d'acteurs («Akteurkonstellationen») se repère pareillement dans les deux ouvrages allemands. Ainsi, Hellmuth Lange y voit-il l'un des trois défis d'une durabilité qui emporte un «changement radical» ("als radikaler Wandel»): «Es bedarf hinreichend breiter, handlungsfähiger und handlungsbereiter Akteurkonstellationen» (p. 37). En tant qu'exemplifications, l'ouvrage déploie une focale à l'échelon des individus, autour de la consommation durable, et une autre sur les problématiques organisationnelles des entreprises et des institutions (partie II: Konsum, et partie III: Unternehmen/Organisation: p. 43-147 et 149-235). Le manuel Nachhaltigkeit dédie également un chapitre aux «Nicht-staatliche Akteure: Unternehmen, Konsumenten, Zivilgesellschaft» (p. 182-203), en lien avec les échelles de concrétisation de la durabilité: locale (Agendas 21 comme exemple), nationale en Allemagne, européenne, Nations unies et modèle de la "gouvernance globale». Plus loin, on note aussi la question de l'établissement d'une «culture de la durabilité» («Kultur der Nachhaltigkeit», p. 229 sq.), c'est-à-dire la transformation des pratiques individuelles et les modes d'imposition sous-jacents, lorsqu'on évoque l'éducation au développement durable.

\section{L'articulation entre approches substantielles et procédurales}

Troisième caractéristique partagée, la durabilité est analysée via une double entrée contenus/procédures. C'est là un résultat marquant, tant dans l'appréhension de ce qui se joue derrière l'affirmation du développement durable (et comment, dans quel cadre) que pour relativiser la lecture du compromis entre les «trois volets». Du reste, la dimension procédurale de la mise en œuvre du développement durable fait aussi l'objet d'une insistance dans les manuels de sociologie de l'environnement précédemment cités. Ainsi, dans Environnement et société, il est clairement soulevé qu' «à l'échelle locale, le développement durable se décline par la multiplication de procédures de normalisation. [...] La quête de légitimité dans l'application locale des principes internationaux et surtout dans l'articulation entre les deux niveaux constitue le lieu de positionnement actuel pour une partie des sciences sociales» (p. 102-103) - ce que confirme le chapitre «Développement durable et gouvernance urbaine, perspectives croisées" dans le Manuel de sociologie de l'environnement.

On lit en ce sens les parties I à IV du Handbook dirigé par Bertrand Zuindeau, à travers, d'une part, les «enjeux territoriaux» (partie III, par exemple ayant trait à la ville, la ruralité, la biodiversité, etc.) et les «enjeux sectoriels» (partie IV, traitant de la pollution atmosphérique, du changement climatique, de l'eau, des déchets, de la 
santé, de l'agriculture, du tourisme, de la construction et des transports), et, d'autre part, les «concepts et méthodes» (partie I) et les "problématiques générales» (partie II), où sont étudiées les dimensions processuelles et procédurales, à l'exemple notamment de «l'évaluation des politiques territoriales au regard du développement durable» (p. 71 sq.), des «indicateurs de développement durable à l'échelle des territoires» (p. 83 sq.) et des «innovations environnementales» (p. 143 sq.) - ou encore les deux derniers chapitres de l'ouvrage: «Développement durable et "bonnes pratiques" » (p. 479 sq.) et "La gouvernance, une impérieuse nécessité pour le développement durable» (p. 491 sq.). Développement durable, communautés et sociétés va dans le même sens, en soulignant par exemple les jeux sur les normes et les règles, c'est-à-dire sur les cadres et modes d'intervention en développement durable («Développement durable urbain et transactions territoriales», p. 157-170) plutôt que sur d'éventuels secteurs clefs, tant dans le domaine de l'agriculture que de la ville, qui sont les deux grandes entrées explorées dans le livre.

Nachhaltigkeit propose également, à travers les chapitres 5 et 6 , une mise en parallèle des principaux domaines d'application de la durabilité, c'est-à-dire sa dimension substantielle: "Gesellschaftliche Handlungsfelder» (chap. 6, p. 107 sq.), où sont déclinés : Ernährung, Wohnen und Bauen, Mobilität, Energie, Klimawandel, Wasser, Arbeit, Landwirtschaft; en regard du chap. 5: «Nachhaltige Entwicklung konkret: messen, bewerten, handeln» (p. 76 sq.), où il en va des enjeux processuels et procéduraux (notamment en lien avec les indicateurs, la question de la mesure et de l'évaluation, les modèles et scénarios...), ainsi que de la section 3 du chapitre 3 où est traitée la dimension démocratique qui se veut rattachée à la durabilité (p. 40 sq.). Enfin, Nachhaltigkeit als radikaler Wandel pose clairement cette hypothèse de départ: «Die Nachhaltigkeitsproblematik wird [...] zu einer Herausforderung im Sinne weiterer Schritte zur Modernisierung und Entfaltung der Demokratie, und dies sowohl in prozeduraler als auch inhaltlicher Hinsicht» (p. 12, c'est nous qui soulignons). C'est en cela - «inhaltliche und prozessuale Nachjustierungen» (p. 37) - que se comprend le troisième défi dégagé par Hellmuth Lange: «Es muss gelingen, sowohl die Zielstellungen als auch die notwendigen Akteurkonstellationen trotz des unvermeidlichen und nicht endenden Wandels des Kontextbedingungen immer wieder zu rekonfigurieren » (p. 37). Le livre interroge ainsi la problématique de la gouvernance, avec par exemple les questionnements du changement socio-technique et de la place des ONG ( Soziotechnischer Wandel», «Nichtregierungsorganisationen als "Player" in der Nachhaltigkeitspolitik», p. 237-341).

\section{Des dynamiques processuelles et transactionnelles permanentes}

La durabilité est également qualifiée dans les ouvrages retenus, en France et en Allemagne, comme relevant d'une dynamique processuelle, caractérisée plus précisément par deux grandes propriétés. D’une part, elle se marque dans des expérimentations toujours en train de se faire (et non pas un état figé ou abouti). Comme l'exprime Hellmuth Lange, «es erscheint angemessener und fruchtbarer [...] nicht nach Nachhaltigkeit schlechthin zu suchen, sondern die Bedingungen für mehr oder weniger radikale Veränderungen zugunsten von nachhaltigeren Optionen der Entwicklung in den Mittelpunkt zu stellen» (p. 10). Cette idée se retrouve dans le chapitre 5 de l'ouvrage Nachhaltigkeit: «Nachhaltige Entwicklung konkret: messen, bewerten, handeln» (p. 76 sq.), 
c'est-à-dire la dimension processuelle de mise en œuvre et d'évaluation de la durabilité. De même, dans son introduction à Développement durable et territoire, Bertrand Zuindeau souligne:

«Une approche territoriale orientée vers la compétitivité et l’attractivité ne garderait sa légitimité que si elle intègre des considérations relatives au maintien des ressources, à la gestion des risques, ou à la recherche d'équité sociale. Sur un plan de mise en œuvre des politiques, une telle caractérisation tient plus actuellement de la perspective souhaitable que de la réalité observable. Sa concrétisation ne paraît guère pouvoir se faire autrement que progressivement, et l'orientation proprement durable de la politique est peut-être même à rechercher dans une démarche in itinere ("chemin faisant") plutôt que définie en amont, une fois pour toutes» (p. 15).

Il est ainsi question, au fil des parties de l'ouvrage, d'«apprécier les aspects territoriaux des voies de solutions proposées, concernant notamment les gouvernances présentes ou à constituer» (p. 19-20).

D'autre part, la durabilité prend place in concreto dans des dynamiques incrémentales, par essais et erreurs, en particulier à des échelles réduites, et via des transactions sociales, qui sont au cœur des contributions à l'ouvrage Développement durable, communautés et sociétés - en particulier les chapitres «Au cœur du développement durable démocratique. Transactions sociales entre individus, communautés et sociétés» (p. 33-46), rédigé par Maurice Blanc, et «Développement durable urbain et transactions territoriales» (p. 157-170), où sont dépliées, suivant un double balancement, les transactions sur les intérêts et sur les valeurs, puis les transactions sur les principes légitimes et sur les règles, dont les cheminements de concrétisation caractérisent des configurations sociospatiales et des espaces de références. Ceci rejoint le concept d'«échec fructueux» («erfolgreiches Scheitern») que met en avant Hellmuth Lange dans Nachhaltigkeit als radikaler Wandel (p. 37) à partir des travaux de Johannes Weyer ${ }^{(45)}$.

5. Faire preuve de réflexivité: aborder ce qui peut être à la fois une notion sociétale et un concept scientifique

À un cinquième niveau, qu'en est-il alors de la durabilité en termes épistémologiques $^{(46)}$ ? Des ouvrages considérés, elle apparaît constituer à la fois un objet d'étude et un cadre analytique pour saisir des transformations sociétales contemporaines. Objet, car la durabilité, on l'a dit, a un certain contenu repérable, qui renvoie en même temps à des cheminements et des configurations. Analyseur, car elle emporte indissociablement aussi un mode d'intelligibilité du réel, donne à voir des références et des ressources et permet de relire des dynamiques d'action, leur production comme leur opérationnalisation. En cela, le répertoire de la durabilité a une double portée instrumentale et heuristique, et c'est bien ce que traduit Développement durable et territoire au fil de ses 40 chapitres; Bertrand Zuindeau note dans son introduction: "Si [...] les contenus sont plutôt de nature positive, certains développements sont, toutefois, marqués par une certaine normativité. C’est notamment le cas du dernier chapitre

45 Johannes WEYER, «System und Akteur. Zum Nutzen zweier soziologischer Paradigmen bei der Erklärung erfolgreichen Scheiterns», Kölner Zeitschrift für Soziologie und Sozialpsychologie, 45/1 (1993), p. 1-22.

46 Voir également P. Hamman, Sociologie urbaine (note 2), p. 44-54. 
relatif à la gouvernance, qui offre des perspectives pour l'avenir» (p. 17). Précisément, la partie I "Concepts et méthodes" vise à répondre à des enjeux de recherche que l'on peut soulever par rapport au statut de l'objet développement durable: «Répondre aux exigences épistémologiques requises; Élaborer des méthodes adaptées; Étudier de manière critique les méthodes existantes" (p. 19).

On comprend dans le même sens les essais de théorisation produits en sciences sociales au cours de la décennie 2000, qui qualifient la durabilité d'utopie performative. Tout en validant, durant ces années de diffusion de la notion, l'hypothèse d'une «nouveauté» de la durabilité comme paradigme, ce sont également l'opérationnalité et les usages de ce registre qui se retraduisent dans les ouvrages considérés. Particulièrement parlant est Développement durable, communautés et sociétés, où il est question, sous la plume de deux coordinatrices, de «l'utopie mobilisatrice du développement durable» (Josiane Stoessel-Ritz, p. 9-16) ${ }^{(47)}$ et d'un point réflexif sur «l'utopie du développement durable et la recherche urbaine» (Nicole Mathieu, p. 199-219).

En effet, la durabilité est d'abord une notion sociétale et politique, plus qu'un outillage scientifique, ainsi que le remarque Hellmuth Lange: "Trotz des starken Bezuges der Nachhaltigkeitsthematik auf wissenschaftliche Fakten (vor allem naturwissenschaftlicher, aber auch gesellschaftswissenschaftlicher Art) bleibt sie eine regulative Idee und bei entsprechender Nutzung ein politisch-gesellschaftliches Reflexionsinstrument» (p. 10). L'auteur avance comme brique de base dans le chapitre théorique d'introduction: "Nachhaltigkeit ist nur sehr bedingt ein wissenschaftliches Konzept. Zwar hat die Expertise von Wissenschaftlern im Entstehungsprozess des Nachhaltigkeitskonzepts eine nennenswerte Rolle gespielt. Tatsächlich gehören aber die Agenda 21 und das Konzept der Nachhaltigkeit vor allem anderen in den Bereich der Politik» (p. 13, souligné par l'auteur). La durabilité est donc considérée comme un énoncé de portée politique; Hellmuth Lange évoque la nécessité de faire de la durabilité le pivot de toute politique (p. 37). Ceci montre bien l'importance, de plus en plus établie dans la littérature à partir de la deuxième moitié des années 2000, d'une mise à distance des fausses évidences d'une durabilité parfois avancée comme un bien commun partagé. De là se conçoit aussi une dimension critique, à l'instar du chapitre 10, «Rezeption und Kritik» (p. 219 sq.) du manuel Nachhaltigkeit. Ces lectures critiques prennent, de façon générale, une place croissante à la suite de la crise économique mondiale de 2007-2008, jusqu'à poser le questionnement d'alternatives au paradigme de la durabilité.

\section{Des alternatives à la durabilité? L'exemple du répertoire de la décroissance/Postwachstum}

Ce questionnement ressort plus nettement ces dernières années, mais de façon diverse, dans les études de sciences sociales. Ainsi que nous l'avons montré à partir d'un corpus de revues scientifiques francophones et anglophones depuis 2009 autour du marqueur de la «ville durable», on voit apparaître, dans des proportions qui restent limitées par rapport aux énoncés de la durabilité, quatre principales «alternatives»:

47 Ceci n'est pas sans référence au «réalisme utopique» dont parle Anthony Giddens, Les conséquences de la modernité, Paris, L'Harmattan, 1994, p. 160. 
« décroissance», «résilience», «transformation durable» et «transition », qui s'avèrent toujours largement enserrées dans une vision binaire (pour/contre) par rapport à la croissance économique comme référent central ${ }^{(48)}$. Il y a là tout un univers de références - et non un corpus unifié ${ }^{(49)}$ - qu'il serait vain de prétendre totalement cerner, d'autant qu'il faut tenir compte du fait que les ouvrages rédigés sur ces enjeux le sont généralement par des auteurs eux-mêmes «engagés» et publiant auprès de maisons d'édition elles aussi identifiées. En termes de circulations en France et en Allemagne, nous nous arrêtons sur le registre de la décroissance comme indicateur permettant de formuler cinq remarques:

1. Significativement, c'est depuis la crise économique de 2007-2008 que les débats sur la décroissance ont (re)pris de l'ampleur, tant en France qu'en Allemagne. Wachstum - Krise und Kritik, du collectif AK Postwachstum, publié en 2016, se positionne clairement par rapport à ce nouveau contexte:

"Seit Beginn des 21. Jahrhunderts setzen sich Menschen für "Décroissance", "Degrowth" oder "Postwachstum" ein, und seit der Krise 2008 wird diskutiert, ob die kapitalistische Weltwirtschaft in eine "säkulare" Stagnation geraten ist. Die Debatte um die Grenzen des Wachstums ist als Kritik des globalen Kapitalismus wieder aufgeflammt. Dieser Band bietet neue Perspektiven: Er diskutiert, ob der Kapitalismus weltweit an seine Wachstumsgrenzen geraten ist; er stellt Alternativen neben- und gegeneinander; schließlich fragt er, wie der Weg in eine nicht mehr von Wachstum abhängige Gesellschaft demokratisch gestaltbar wäre» (présentation de l'ouvrage).

Le discours est similaire côté français, où Denis Bayon écrit dans sa postface à l'édition de 2012 de La décroissance: «Deux ans après la publication de ce livre, le débat sur la question de la décroissance n'a cessé de prendre de l'ampleur. [...] L’effondrement de l'économie d'accumulation n'y est sans doute pas pour rien» (p. 235). Si l'on suit Stéphane Lavignotte dans La décroissance est-elle souhaitable?, il y aurait là l'incarnation d'un retour militant à certaines références énoncées dans les années 1970, une fois le paradigme du développement durable capté par les institutions dominantes et ses discours de compatibilité entre croissance économique, attention écologique et progrès social démentis par la crise. Au-delà de la posture de l'auteur lui-même, ceci appuie l'hypothèse du développement durable comme énonciation contingente rattachée aux décennies 1990-2000:

«Trente-sept ans après son utilisation par André Gorz ${ }^{(50)}$ à l'occasion du rapport du Club de Rome sur les limites de la croissance, le terme de décroissance a fait un retour fracassant. [...] Le "développement durable", popularisé au sommet de la Terre à Rio en 1992, a été récupéré par les grandes entreprises comme par les partis politiques classiques. [...] Avec le concept de décroissance, l'écologie politique s'offre une seconde jeunesse subversive. Les auteurs de la décroissance revisitent les "fondamentaux" de l'écologie politique et radicalisent ses thèmes. Une mobilisation militante se dessine petit à petit [...]» (p. 11).

48 P. Hamman, «Definitions and Redefinitions of Urban Sustainability» (note 23).

49 Denis Bayon, Fabrice Flipo et François Schneider parlent eux-mêmes de "la galaxie "décroissance" " dans l'introduction de l'ouvrage La décroissance (p. 8-11), et d'une "vaste mouvance hétéroclite» (p. 21).

50 Référence à André Gorz, Critique du capitalisme quotidien, Paris, Galilée, 1973. 
2. Dès lors, et tout comme la durabilité est un énoncé proprement politique en même temps qu'une notion voire une grille d'analyse, la décroissance se lit indissociablement sous le double statut d'un concept et d'un mouvement. Comme l'exprime Stéphane Lavignotte dans La décroissance est-elle souhaitable? :

«D’un concept [...] relisant les classiques de l'écologie politique, croisant les travaux plus anciens de Serge Latouche ${ }^{(51)}$ et ceux critiquant la "mal-bouffe" de Paul Ariès ${ }^{(52)}$, la décroissance est devenue un mouvement, renouant avec l'écologie comme mouvement - et pas seulement parti - au moment où reprennent de la vigueur le mouvement antinucléaire, l'agriculture paysanne et biologique ou la contestation de la voiture en ville. Des pratiques originales, un programme, un travail idéologique de démarcation et une entrée dans le débat politique...» (p. 39).

C'est bien ce que souligne aussi Marc Hieronimus dans Der Schritt zur Seite, rattachant le mouvement de la décroissance à une genèse française, celle des années 1970 et de Mai 68 («Ursprünge im Pariser Mai 1968»).

3. Il y a là trace de circulations notionnelles entre les deux cadres nationaux, dont une marque - comme pour le développement durable/soutenable - se repère aussi quant aux enjeux de traduction. Significativement, le livre de Marc Hieronimus s'intitule: Der Schritt zur Seite: Postwachstum - Rückgang - Décroissance, et l'auteur utilise largement le terme français de décroissance, expliquant p. 9: "Es gibt bislang keine schöne Übersetzungen. Die vorgeschlagenen Wörter "Negativ-" und "Postwachstum" haben noch zu sehr das Wachstum als Bezugspunkt. » Ceci dit, là où certains concepts associés, comme celui de convivialité, apparaissent directement repris ${ }^{(53)}$, il est plus souvent question de «l'après-croissance» ou Postwachstum en Allemagne, tandis que le terme de décroissance est davantage usité en France. Mais ce constat n'est pas unique; ainsi, dans l'ouvrage du collectif AK Postwachstum, la partie V s'intitule «Degrowth und Demokratie» et la partie IV portant sur les acteurs de la transformation comprend deux chapitres qui utilisent le terme degrowth et non Postwachstum: «Transformationspotenziale der Degrowth-Bewegung» et "Degrowth und die Frage des Subjekts». De plus, si l'on voit dans ces mots certaines nuances sémantiques, on peut aussi opérer un rapprochement en référence à la formulation d'«a-croissance» proposée par Serge Latouche:

"Le mot d'ordre de décroissance a ainsi surtout pour objet de marquer fortement l'abandon de l'objectif de la croissance pour la croissance, objectif dont le moteur n'est autre que la recherche du profit par les détenteurs du capital et dont les conséquences sont désastreuses pour l'environnement. En toute rigueur, il conviendrait de parler d'a-croissance comme on parle d'a-théisme, plutôt que de dé-croissance. C'est d'ailleurs très précisément de l'abandon d'une foi ou d'une religion qu'il s'agit: celle de l'économie, de la croissance, du progrès et du développement ${ }^{(54)}$.

51 Par exemple, Serge Latouche, La planète des naufragés. Essai sur l'après-développement (1991), Paris, La Découverte, 2016.

52 Notamment Paul Ariès, Les Fils de MacDo, Paris, L'Harmattan, 1997.

53 Voir notamment Caillé/Humbert/Latouche/Viveret, De la convivialité (note 29); Adloff/ HeIns, Konvivialismus (note 29).

54 Serge Latouche, Le pari de la décroissance, Paris, Fayard, 2006, p. 4. 
D’emblée, l'ouvrage La décroissance invite également à ne pas en rester à un fétichisme des expressions, et se positionne en termes de processus - face à celui incarné par le développement durable - et de réception sociale:

«La décroissance n'est pas la fin de l'histoire, l'état actuel du débat est amené à évoluer - le mot "décroissance" disparaîtra peut-être une fois accompli son rôle de réarmement de l'activité critique. [...] Son argument principal est bien celui de l'innovation: la décroissance n'est peut-être pas la solution à tout, mais elle ose bouleverser les clivages hérités, pour les mettre en question» (p. 18-19).

4. La montée en puissance du répertoire de la décroissance s'incarne désormais dans des ouvrages organisés comme des tentatives de synthèse «impliquées», ce qui marque aussi une étape en termes de diffusion. Ceci se repère dans les résumés de chacun des quatre ouvrages considérés, aussi bien celui de Marc Hieronimus: «Das Buch stellt die Motive, Denker und falschen Freunde der französischen Wachstumskritiker vor. Es ist die erste deutschsprachige Gesamtdarstellung der Décroissance-Bewegung»; que celui du collectif AK Postwachstum, dont l'éditeur met en exergue le commentaire suivant: «Seminare zum Thema bekommen mit dem Band eine fast vollständige Literaturbasis an die Hand »; ou encore celui de Stéphane Lavignotte, qui s'affiche en $4^{\text {e }}$ de couverture comme « un panorama guidé par une sympathie critique». Enfin, l'ouvrage de Denis Bayon, Fabrice Flipo et François Schneider, La décroissance, sous-titré Dix questions pour comprendre et débattre, se veut pédagogique et accessible, de diffusion élargie, en répondant à des questions courantes - par exemple: «La décroissance, une idée neuve ou une vieille idée réactionnaire?", "Pourquoi la décroissance et pas le "développement durable" ?», «La décroissance, est-ce la fin du progrès scientifique et technique?», etc.

5. Les ouvrages de synthèse étudiés se rejoignent autour d'une définition de la décroissance autour de sept déclinaisons principales, qui percolent en France et en Allemagne:

(i) Une critique - à la fois sociale et écologique - du capitalisme et de la société industrielle, vus comme un problème structurel, tel que le pointent le collectif AK Postwachstum (Wachstum - Krise und Kritik, partie I) et Marc Hieronimus (chap. 2 et 3 : «Warum Décroissance? » et «Was hindert uns?»). C’est aussi le sens du premier chapitre de La décroissance, où les auteurs expliquent la portée critique de la notion, en mobilisant différents courants de pensée: «de la critique écologique à la critique du système des besoins", "de l'entropie à la critique de l'économie politique», "de la critique démocratique à la critique culturaliste», pour évoquer enfin une «crise de sens» (p. 21-60). De même, dans le premier chapitre de La décroissance est-elle souhaitable?, Stéphane Lavignotte expose «le retour d'une écologie politique radicale» (p. 17 sq.).

(ii) Une réflexion sur les notions de développement et de croissance et les inégalités qui les accompagnent, en particulier les inégalités socio-écologiques et Nord-Sud, ainsi qu'il est développé dans Wachstum - Krise und Kritik (partie II). Dans Der Schritt zur Seite, Marc Hieronimus résume ainsi:

" "Entwicklung" und "Fortschritt" sind also versteckt politische, wenn nicht religiöse Begriffe, auf die hier weitgehend verzichtet werden soll. Sie bedeuten freilich nicht das gleiche. Oft meint "Entwicklung" auch etwas ganz Ungewolltes, etwa die aktuellen Umwelt "entwicklungen", die kaum jemand als Fortschritt bezeichnen wollte. Aber eben: 
Weil wir sowohl ungewollte als auch gemachte, aus dem rational-industriellwirtschaftlichem Treiben mit Namen Fortschritt herrührende Veränderungen mit dem gleichen Wort benennen, ist es zu meiden. Es verwischt die hier so wichtigen Grenzen und ist politisch» (p. 11, c'est l'auteur qui souligne).

Une mise à distance des notions de développement, de croissance et de mondialisation - y compris à la faveur d'une «autre» mondialisation, celle prônée par les mouvements altermondialistes ${ }^{(55)}$ - se retrouve aussi dans l'ouvrage La décroissance, y compris quant aux inégalités Nord-Sud, aux chapitres 3, 7 et 8 :

«Pourquoi la décroissance et pas le "développement durable" ? Où il est utile de rappeler le sens d'une expression dont il est fait usage de façon fréquente et inconsidérée» (p. 81 sq.); «La décroissance, c'est la récession, le chômage, la fin de l'économie de marché? Où il est montré qu'il est impudent d'accuser les dissidents de vouloir propager les maux distillés par le système qu'ils critiquent» (p. 163 sq.); «La décroissance concerne-t-elle les pays du Sud? Où il apparaît que la mondialisation montrerait une bien meilleure figure si l'“Occident" prenait au sérieux les choix de vie des "sous-développés" (p. 189 sq.).

Stéphane Lavignotte évoque à son tour «une critique de la croissance et de la consommation comme néfastes à la planète... qui s'élargit à une critique de la richesse comme néfaste au bonheur des personnes », c'est-à-dire une "reprise des critiques de l'écologie politique historique», à laquelle s'adjoint «un apport original et polémique face à la banalisation du discours écologiste", soit une prise de position "contre le développement durable et le capitalisme vert». Cette dernière est appuyée sur «une critique de l'économie dominante avec Nicolas Georgescu-Roegen" et passe par "un débat fratricide avec les tenants d'“un autre développement possible"» (p. 19-38), tout en interrogeant le «sens ou non-sens écologique et social d'une baisse du PIB» (p. 53-57) et «la prise en compte des inégalités et de la structure de classe» dans les réflexions en termes de décroissance (p. 61-72).

(iii) Une critique de la croyance en une solution technologique, en se situant après le machinisme: "nach dem Machinensturm ", pour reprendre le titre d'un chapitre de Wachstum - Krise und Kritik (p. 159 sq.). De façon proche, Stéphane Lavignotte souligne « une reprise de la critique ellulienne de la technique» (p. 25-27) ${ }^{(56)}$. En même temps, il s'agit aussi d'écarter une autre critique, celle-là adressée à l'encontre de la décroissance, à savoir de prôner un retour en arrière vers un mode de vie "primitif». Marc Hieronimus écrit ainsi: «Rückgang also, aber bis wohin? Nein, DécroissanceAnhänger wollen nicht zurück in die Höhle oder auf den Baum, nicht einmal nur in die vorelektrische Zeit, obwohl sie einiges für sich hatte» (p. 13). Et on lit ironiquement dans l'ouvrage La décroissance: "La décroissance, est-ce la fin du progrès scientifique et technique? Où il apparaît que le retour aux cavernes humides et aux chandelles n'est pas précisément le modèle en vogue, même chez les radicaux irresponsables » (chap. 4, p. 103 sq.).

55 Par exemple, Denis Bayon, Fabrice Flipo et François Schneider mettent en avant, pour les pays du Sud, la possibilité de stratégies économiques de "relocalisation» et celle d'y réintroduire de la diversité par rapport à ce qu'ils considèrent comme l'uniformisation marchande occidentale, en mobilisant plusieurs points de vue dans la littérature «décroissante», La décroissance (note 15), p. 203. 
(iv) Des pistes et des bases pour fonder une vision sociétale alternative. C'est le sens du titre de l'ouvrage Der Schritt zur Seite, qui pose de suite en introduction: "Lassen wir uns nicht einreden, die Welt sei, wie sie ist, weil alles was kam, so habe kommen müssen. Es gab und gibt immer Alternativen» (p. 10). C’est aussi ce qui explique, plus largement, une double posture des développements: en intégrant un corpus de textes et d'auteurs reconnus désormais comme «classiques» de la pensée de la décroissance (chapitres 1 à 5, avec notamment un focus sur «Vor- und Nachdenker der Décroissance» et "Falsche Freunde der Décroissance»), et en tant que propos orienté vers l'action, la transformation concrète de la société (chapitres 6 à 8 : «Der Weg der Décroissance», "Konkrete Vorschläge», «Die Zukunft»). De même, au sein de l'ouvrage Wachstum - Krise und Kritik, il est question d'ouvrir des pistes: «Der Ausbau von Dienstleistungen als Grundlage einer Postwachstumsgesellschaft» (p. 115 sq.) et «Postwachstumsökonomik als Reduktionsprogramm für industrielle Versorgungssysteme» (p. 135 sq.). Le dernier chapitre de La décroissance cherche aussi à se projeter, en posant la question : «que signifierait concrètement une politique de décroissance?», et en soulignant transversalement «une critique de la technique à visée universaliste» mais aussi des divergences entre différents courants sur ce «à quoi pourrait ressembler un programme politique de décroissance» (p. 225-234). Stéphane Lavignotte évoque également la «simplicité volontaire» comme "pratique» et «la relocalisation et la promotion de l'usage» comme "programme» (p. 39-45).

(v) Les acteurs en jeu, notamment quant à l'organisation du mouvement de la décroissance ou encore des "Commons \& Care» (Wachstum - Krise und Kritik, partie IV), ainsi que les porteurs du mouvement, ou les relectures et interprétations qui en sont faites (Der Schritt zur Seite, chap. 4 et 5: «Vor- und Nachdenker der Décroissance» et "Falsche Freunde der Décroissance»; chap. 1 de La décroissance; ainsi que La décroissance est-elle souhaitable?, qui traite sous l'angle des acteurs aussi bien la présentation du paradigme de la décroissance que les débats et critiques qu'il suscite, et sa place dans le paysage politique français, en particulier à gauche).

(vi) Le rapport entre décroissance et démocratie, incarné:

a. dans une relation renouvelée aux innovations sociales: "Vorwärts in die Vergangenheit», énonce un chapitre du livre Wachstum - Krise und Kritik (p. 245 sq.) ${ }^{(57)}$. La décroissance est alors bien définie comme une vision innovante: «La décroissance, une idée neuve ou une vieille idée réactionnaire? Où l'on montre que, dans un monde nouveau et souvent inquiétant, certains ne se contentent pas des vieilles formules réchauffées" (La décroissance, chap. 2, p. 61 sq.).

b. en redéfinissant une démocratie radicale («radicale Demokratie») (Wachstum - Krise und Kritik, partie V). Denis Bayon, Fabrice Flipo et François Schneider prennent soin de déminer la question: «La décroissance n’implique-t-elle pas

57 Quant à Marc Hieronimus, il pointe en exergue de son ouvrage ce retour aux réflexions/utopies des années 1970: "Alles anhalten. Nachdenken. Und das ist keine traurige Angelegenheit”. So lautete das Motto einer französischen Comic-Kolumne der Siebziger Jahre, als weltweit die letzten Utopien geschrieben wurden. [...] Die französische Décroissance-Bewegung greift dagegen Kritik und Utopie von damals wieder auf: Weniger Arbeit, Besitz, Konsum bedeuten ein Mehr an Freundschaft, Freizeit und Genuss », Der Schritt zur Seite (note 16). 
une vision dirigiste ou autoritaire de la politique?» (chap. 9, p. 205 sq.). Stéphane Lavignotte propose aussi une focale sur les tenants de la décroissance face à l'exercice de représentation démocratique, et notamment le rapport aux forces politiques de gauche. Il met ainsi en avant « des interpellations souhaitables pour les gauches»: «Décoloniser l'imaginaire productiviste des gauches», «La révolution politique de la valeur d(e) (l')usage» et "Pour une relecture de l'héritage des Lumières » (chap. 3, p. 83 sq.) ; et appelle au final: «Contre toutes les pensées à majuscule, il faut encore compiler et tenter de penser ensemble la pluralité des positions politiques: une composition politique à imaginer [...]»(p. 120, souligné par l'auteur). Autrement dit, il convient de se méfier d'une «pédagogie des catastrophes» qui risquerait de faire le lit de l'autoritarisme (p. 95).

(vii) Enfin, l'idée, posée avec insistance, d'une décroissance heureuse. Marc Hieronimus résume: «Rückgang/Décroissance bedeutet: Drehen wir nicht an Rädchen, halten wir die Maschine an - und das ganze mit Freude!» (p. 12); et plus loin : "Wovon wollen wir mehr? Unterhaltung, Kontrolle und Psychopharmaka, oder von Gespräch, Freiheit und Festlichkeit?» (p. 17). De même, le chapitre 6 de l'ouvrage La décroissance développe cette question: "privation ou joie de vivre? Où l'on se demande sérieusement si les habitants des États-Unis sont vraiment plus heureux que ceux du Bhoutan» (p. 139 sq.), soit une «critique du "bonheur conforme" " (p. 140) - ce que Stéphane Lavignotte pointe aussi à travers une critique de la richesse comme néfaste au bonheur des personnes (p. 22-25). Il est alors question de «simplicité volontaire» et de «sobriété heureuse» - au sens de Pierre Rabhi ${ }^{(58)}$, du reste fréquemment cité (par exemple, dans La décroissance, p. 56) -, c'est-à-dire du lien avec une éthique et certains modes de vi(vr) $\mathrm{e}^{(59)}$.

\section{Conclusion}

Ainsi qu'il ressort des manuels et ouvrages de référence étudiés, le développement durable s'apparente, en France comme en Allemagne, à un concept à la fois communément diffusé et approprié, et contingent ou transitoire, c'est-à-dire dont la pertinence pratique a été avérée, durant les décennies 1990 et 2000, en modifiant le référentiel de l'action publique et en s'imposant comme objet des sciences sociales. La notion s'est développée durant des années de mondialisation, dont le contexte a changé depuis la crise de 2007-2008, ce qui explique des débats sur des visions alternatives de la société plus visibles depuis la fin des années 2000 .

Au final, en termes de circulations, cet article met à distance plusieurs fausses évidences. Clairement, il n'y a pas de processus linéaire ou «qui va de soi» en matière de durabilité ou encore de conscience écologique. Si bien qu'il n'y a pas non plus ni modèle unique - pas plus en France qu'en Allemagne, et, y compris au sein des milieux «alternatifs", il y a nombre de débats et de divergences - ni position nationale "en pointe» ou «en retard»: les visions duales (dont durabilité forte versus faible) sont toujours à relativiser en fonction des secteurs d'intervention, des critères en jeu, etc., et à rapporter à des dynamiques marquées par des emprunts et des modes de diffusion

58 Pierre Raвнi, Vers la sobriété heureuse, Paris, Actes Sud, 2010.

59 Voir sur ce point Aurélie Choné, «Ecospirituality», in: Choné/Hajek/Hamman, Rethinking Nature (note 43 ), p. 38-48. 
qui sont autant de filtres. Pour preuve, là où l'on associe volontiers l'Allemagne à des réalisations innovantes (à l'instar d'éco-quartiers "modèles» comme à Fribourg-enBrisgau...) et à une intériorisation des enjeux de durabilité plus forte qu'en France parmi la population (qu'incarnerait par exemple le succès politique du parti des Verts/ Die Grünen), on s'aperçoit que des références au mouvement de la décroissance, vu comme précurseur, percolent en la matière en Allemagne... à partir des milieux intellectuels français des années 1970. Des enjeux de traduction (le terme décroissance utilisé aussi en allemand...) et d'hybridation des concepts (Postwachstum et a-croissance...) s'ensuivent, qui complexifient l'appréhension des appropriations: qu'est-ce qu'être pionnier, pour et reconnu par qui, et avec quel contenu? Des passages indirects se comprennent également de la sorte, à l'exemple de la notion de «modernisation écologique», qui ne s'est pas tant diffusée directement d'Allemagne en France - malgré les travaux fondateurs de Joseph Huber -, que par le truchement de chercheurs néerlandais. Des questions linguistiques se retrouvent ici, l'internationalisation s'étant avérée de facto facilitée par l'usage véhiculaire de l'anglais.

Cette dernière remarque rejoint notre hypothèse de départ: ne pas considérer, lorsqu'il est question de manuels, la sphère scientifique en sciences sociales comme hermétique aux pratiques sociales, mais comme un écho - indirect là encore, à travers les protocoles d'objectivation. Sur ce plan, deux dynamiques interreliées apparaissent constitutives des savoirs de la «durabilité» en France et en Allemagne. L'une est réflexive, en tant que corpus de connaissances, où il s'agit d'inventer de nouveaux cadres de pensée pour saisir les interactions environnement/société dans leur dimension relationnelle. L'autre est principe d'action, à visée d'intervention, y compris à travers les recompositions d'énoncés et d'objets disciplinaires: cette démarche plus opératoire renvoie à un «engagement dans le monde», qui vise à faire émerger de nouvelles formes de vivre ensemble et d'agir sur le rapport des sociétés à l'environnement. C'est bien cette double entrée qui permet de lire la durabilité sous l'angle des Humanités environnementales, dont il s'agit d'une propriété caractéristique ${ }^{(60)}$.

\section{Résumé}

Cet article interroge les savoirs de la "durabilité» en France et en Allemagne, vus des sciences sociales. Considérant que les travaux et les controverses en sciences humaines et sociales sont des indicateurs valables non seulement d'une actualité de la recherche, mais aussi de débats sociétaux dont ils se font l'écho, il s'appuie sur des lectures croisées de manuels et d'ouvrages saillants allemands et français publiés depuis les années 2000.

La démonstration est conduite en trois temps, examinant (1) en quoi le répertoire de la durabilité est "acté» en France et en Allemagne comme un objet pour des disciplines académiques, à travers l'exemple de manuels de sociologie de l'environnement; (2) ce que désigne plus précisément ce registre en tant que tel au sein des analyses de sciences sociales dans les deux pays, à travers des ouvrages significatifs; et (3) le développement, après la

60 Ainsi qu'il ressort de recherches récentes: Aurélie Choné, Isabelle Hajek et Philippe Hamman (dir.), Guide des Humanités environnementales, Villeneuve d'Ascq, Presses universitaires du Septentrion, 2016; et Choné/Hajek/Hamman, Rethinking Nature (note 43). 
crise économique mondiale de 2007-2008, de répertoires critiques et alternatifs à la durabilité, à commencer par la «décroissance». Ce parcours rappelle qu'il s'agit de questions proprement politiques, et non d'une évidence environnementale «en surplomb».

Au final, en termes de circulations, deux dynamiques corrélatives se dégagent. L'une est réflexive, en tant que corpus de connaissances, où il s'agit d'inventer de nouveaux cadres de pensée pour saisir les interactions environnement/société dans leur dimension relationnelle. L'autre est principe d'action, à visée d'intervention: cette démarche plus opératoire vise à faire émerger de nouvelles formes de vivre ensemble et d'agir sur le rapport des sociétés à l'environnement. C'est cette double entrée qui permet de lire la durabilité sous l'angle des Humanités environnementales, dont il s'agit d'une propriété caractéristique.

\section{Zusammenfassung}

Dieser Artikel hinterfragt die Kenntnisse über die "Nachhaltigkeit“ in Frankreich und Deutschland aus der Sicht der Sozialwissenschaften. Er geht davon aus, dass die Arbeiten und Auseinandersetzungen in den Human- und Sozialwissenschaften als relevante Indikatoren nicht nur für die Aktualität der Forschung betrachtet werden, sondern auch für die gesellschaftlichen Diskussionen, auf die sie sich stützen. Deshalb beruht er auf der vergleichenden Lektüre deutscher und französischer Handbücher sowohl herausragender Werke, die seit den 2000er Jahren veröffentlicht wurden.

Der Nachweis erfolgt in drei Schritten, wobei (1) untersucht wird, inwiefern das Register der Nachhaltigkeit in Frankreich und Deutschland wie ein Gegenstand akademischer Fachgebiete „schriftlich verankert“ ist, zum Beispiel in den Handbüchern der Soziologie und Umweltwissenschaften; (2) was genau dieses Register per se innerhalb der Analysen der Sozialwissenschaften der beiden Länder auszeichnet; und (3) die Entwicklung von Registern nach der Weltwirtschaftskrise von 2007-2008, die der Nachhaltigkeit kritisch gegenüberstehen und Alternativen bieten, angefangen mit der „Décroissance“. Diese Vorgehensweise erinnert daran, dass es sich hier um rein politische Fragen handelt, und nicht um eine „umfassende“, umweltrelevante Evidenz.

Letztendlich kristallisieren sich im Hinblick auf die Wissenszirkulation zwei korrelative Dynamiken heraus. Die eine ist reflexiv, in ihrer Eigenschaft als Wissensgrundlage, wo es darum geht, neue Denkmuster zu entwickeln, um die Interaktionen zwischen Umwelt und Gesellschaft in ihrer relationalen Dimension zu erfassen. Die andere präsentiert sich als Handlungsprinzip, mit dem Ziel, einzugreifen : Diese eher operative Vorgehensweise arbeitet darauf hin, neue Formen des Zusammenlebens entstehen zu lassen und auf die Beziehung der Gesellschaften zur Umwelt einzuwirken. Dieser doppelte Ansatz bietet die Möglichkeit, die Nachhaltigkeit aus dem Blickwinkel der „Environmental Humanities“ $z$ u betrachten, für die sie eine charakteristische Eigenschaft darstellt. 